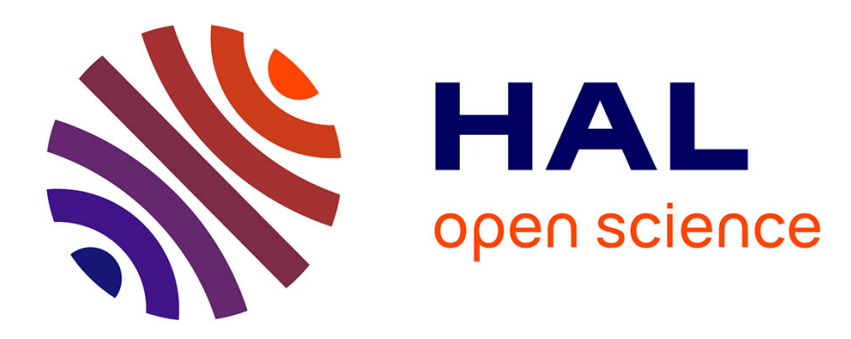

\title{
On shakedown of shape memory alloys structures
}

Michaël Peigney

\section{To cite this version:}

Michaël Peigney. On shakedown of shape memory alloys structures. Annals of Solid and Structural Mechanics, 2014, 6, pp.17 - 28. 10.1007/s12356-014-0035-1 . hal-01093232

\section{HAL Id: hal-01093232 \\ https://hal-enpc.archives-ouvertes.fr/hal-01093232}

Submitted on 10 Dec 2014

HAL is a multi-disciplinary open access archive for the deposit and dissemination of scientific research documents, whether they are published or not. The documents may come from teaching and research institutions in France or abroad, or from public or private research centers.
L'archive ouverte pluridisciplinaire HAL, est destinée au dépôt et à la diffusion de documents scientifiques de niveau recherche, publiés ou non, émanant des établissements d'enseignement et de recherche français ou étrangers, des laboratoires publics ou privés. 


\title{
On shakedown of shape memory alloys structures
}

\author{
Michaël Peigney
}

Received: date / Accepted: date

\begin{abstract}
This paper is concerned with the large-time behaviour of shape-memory alloys structures when they are submitted to a given loading history. Extending the approach introduced by Koiter in plasticity, we state sufficient conditions for the energy dissipation to remain bounded in time, independently on the initial state. Such a behavior is classically referred to as shakedown and is associated with the idea that the evolution becomes elastic in the large-time limit. The study of a particular example shows that the large-time behaviour of shape-memory alloys structures exhibit some complex features which are not found in standard plasticity.
\end{abstract}

Keywords Shape-memory alloys $\cdot$ Shakedown $\cdot$ Sirect methods $\cdot$ Non-smooth mechanics

\section{Introduction}

This paper is concerned with the long-time behaviour of inelastic structures under prescribed loading histories. For elastic perfectly plastic structures, a fundamental result is the Melan's theorem $[14,11]$ which gives a sufficient condition for the energy dissipation to remain bounded with respect to time. That last situation is classically referred to as shakedown, and is associated with the intuitive idea that structure behaves elastically for time $t$ sufficiently large, i.e. that the plastic strain tends to a limit as $t \rightarrow \infty$. Melan's theorem has the distinctive property of being path-independent, i.e. independent on the initial state of the structure. Regarding fatigue design, shakedown corresponds to the most beneficial regime of high-cycle fatigue, as opposed to the regime

Michaël Peigney

Université Paris-Est, Laboratoire Navier (Ecole des Ponts ParisTech, IFSTTAR, CNRS),

F-77455 Marne-la-Vallée Cedex 2, France

E-mail: michael.peigney@polytechnique.org of low-cycle fatigue which typically occurs if the plastic strain does not converge towards a stabilized value [4].

In the particular case of cyclic loadings, it it also known $[9,28]$ that the stress response $\sigma(t)$ converges towards a cyclic response $\sigma_{\infty}(t)$ as $t \longrightarrow+\infty$. Similarly, the rate of plastic strain $\dot{\alpha}(t)$ converges towards a cyclic response $\dot{\alpha}_{\infty}(t)$. Moreover, both $\sigma_{\infty}(t)$ and $\dot{\alpha}_{\infty}(t)$ have the same time pe$\operatorname{riod} T$ as the applied loading. The plastic strain $\alpha(t)$ does not necessarily converge towards a cyclic response, since $\int_{0}^{T} \dot{\alpha}_{\infty}(t) d t$ may be different from 0 . That situation is refered to as ratchetting and implies the collapse of the structure through the accumulation of plastic strain. In the case where $\int_{0}^{T} \dot{\alpha}_{\infty}(t) d t=0$, one classically distinguishes between the cases of shakedown $\left(\dot{\alpha}_{\infty}=0\right)$ and alternating plasticity $\left(\dot{\alpha}_{\infty} \neq\right.$ $0)$. In that last case, the plastic strain $\alpha(t)$ converges towards a cyclic but non constant response $\alpha_{\infty}(t)$. A crucial property of elastic perfectly plastic structures is that the asymptotic rate of plastic strains $\dot{\alpha}_{\infty}$ is unique. This implies that the asymptotic regime (shakedown, alternating plasticity, or ratchetting) is path-independent. That property has fostered the development of direct methods aiming at determining the asymptotic regime for a given cyclic loading, without using a step-by-step incremental analysis [29,1,21,22,13, 25, 27, 24].

All the results mentioned so far apply to elastic perfectly plastic structures, and can be directly extended to the $C$-class of generalized standard materials [10]. Several attempts have been made to extend Melan's theorem to various types of nonlinear behaviour (see [23] for an extensive review). However, as discussed in detail in [23], some of the extensions proposed in the literature lead to non pathindependent results which are therefore of little practical use. This is notably the case for shape memory alloys: shakedown of shape-memory alloys structures has been studied in [5], but the shakedown theorem obtained by those authors has latter been recognized in [23] not to be path-independent. 
The peculiar properties of Shape Memory Alloys (SMAs) - such as the superelastic behaviour or the shape memory effect - are the result of a solid/solid phase transformation between different crystallographic structures (known as austenite and martensite). That phase transformation takes place at the microscopic level and is driven both by thermal and mechanical loading. Much effort has been devoted to developing constitutive laws for describing the behaviour of SMAs. The phase transformation is typically described by an internal variable $\alpha$ which - depending on the complexity of the material model - may be scalar or vectorial. A fundamental observation is that the internal variable $\alpha$ must comply with some a priori inequalities that result from the mass conservation in the phase transformation process. As a consequence, the internal variable $\alpha$ is constrained to take values in a set $\mathscr{K}$ that is not a vectorial space. The presence of such constraints constitutes a crucial difference with plasticity models, and calls for special attention when the structural evolution problem is considered. This last point has been noted in [7] in the context of numerical methods for simulating SMA structures: apart from few exceptions [7, 16, 20,19], most existing numerical methods handle the constraints in an ad hoc fashion, and do not rely on a consistent formulation of the time continuous evolution problem. It has to be observed, however, that mathematically consistent models of evolution problems in shape-memory alloys have been proposed $[6,12]$. One possible approach is to resort to the so-called "non-smooth mechanics" framework (see [6] and references therein), which is not restricted to shape-memory alloys and actually applies in the general situation where constraints are physically imposed on the state variables or their timederivative.

Shakedown theorems in non-smooth mechanics have been recently proposed in [18]. In this paper, we give a direct and self-contained proof of a shakedown theorem for SMA structures. That theorem is used in Sec. 4,5 to study a threebar truss originally considered in [5]. The results in [5] showed that phase-transformation can either increase of decrease the shakedown limit compared to plasticity. Those results, however, are biased by the fact that they are not path-independent. Therefore it is of interest to investigate if such behavior still survives in the context of path-independent shakedown as considered in this paper.

The outline of the paper is as follows. The class of constitutive SMA models that we consider is presented in Sec. 2. A corresponding shakedown theorem is proved in Sec. 3 and compared to the classical Melan theorem in plasticity. The shakedown analysis of the three-bar truss is presented in Sec. 4. We notably discuss the influence of phasetransformation on the shakedown limits (compared to plasticity). Sec. 5 is devoted to cyclic loadings: we use numerical step-by-step analysis to study the asymptotic response of the three-bar truss when the shakedown limit is exceeded.
We show that the asymptotic response for SMAs is more complex than for elastic-plastic materials. In particular, in contrast with plasticity, there is generally no uniqueness of the asymptotic stress rates.

\section{Constitutive laws}

As mentioned in the introduction, SMAs can experience a solid/solid phase transformation between austenite and martensite. The crystallographic structure of the austenite is more symmetric than the crystallographic structure than the martensite. This leads one to distinguish between several martensitic variants corresponding to different orientations of the martensitic lattice with respect to the austenitic lattice. Each martensitic variant is characterized by a transformation strain $\varepsilon_{i}$ that describes the deformation from the austenitic lattice to the martensitic lattice. A simple example is the cubic-totetragonal transformation which is observed for instance in $\mathrm{MnCu}$ and MnTi alloys. In such materials, the austenite has a cubic crystallographic structure (with axes $\left(v_{1}, v_{2}, v_{3}\right)$ ) and the martensitic lattice is obtained by applying a dilatation $\lambda$ along one of the axis $v_{i}$ and a dilatation $\mu$ on $v_{i}^{\perp}$ (Figure 1). There are 3 martensitic variants to be considered, depending on the axis $v_{i}$ along which the dilatation $\lambda$ is applied. Under the assumption of infinitesimal strains, the transformation strains are given by

$\varepsilon_{1}=\left(\begin{array}{lll}\lambda^{\prime} & 0 & 0 \\ 0 & \mu^{\prime} & 0 \\ 0 & 0 & \mu^{\prime}\end{array}\right), \varepsilon_{2}=\left(\begin{array}{lll}\mu^{\prime} & 0 & 0 \\ 0 & \lambda^{\prime} & 0 \\ 0 & 0 & \mu^{\prime}\end{array}\right), \varepsilon_{3}=\left(\begin{array}{lll}\mu^{\prime} & 0 & 0 \\ 0 & \mu^{\prime} & 0 \\ 0 & 0 & \lambda^{\prime}\end{array}\right)$.

with $\lambda^{\prime}=\lambda-1$ and $\mu^{\prime}=\mu-1$. The most commonly used SMAs, such as CuAlNi and NiTi alloys, correspond to cubicto-orthorombic and cubic-to-monoclinic transformations, for which there are respectively 6 and 12 martensitic variants to be considered.

In Fig. 2 is represented a typical uniaxial stress-strain response of SMAs in a strain-driven loading cycle. During the loading, the response is first elastic and subsequently exhibits a stress plateau: the strain increases while the stress remains at a constant value $G^{+}$. In contrast with the elasticperfectly plastic behavior, the plateau is limited: if the imposed strain is sufficiently large, the stress starts to increase again in a linear fashion. At unloading, the response is elastic until the stress reaches a critical value $G^{-}$. There is subsequently a stress plateau, followed by an elastic unloading. No residual strain remains at the end of the loading/unloading cycle. The response in Fig. 2 is typical of the so-called super elastic (or pseudo elastic) response of SMAs, which is observed for sufficiently high temperature.

The physical explanation of the superelastic response in Fig. 2 is the following: at sufficiently high temperature, the material is initially fully austenitic and behaves elastically 


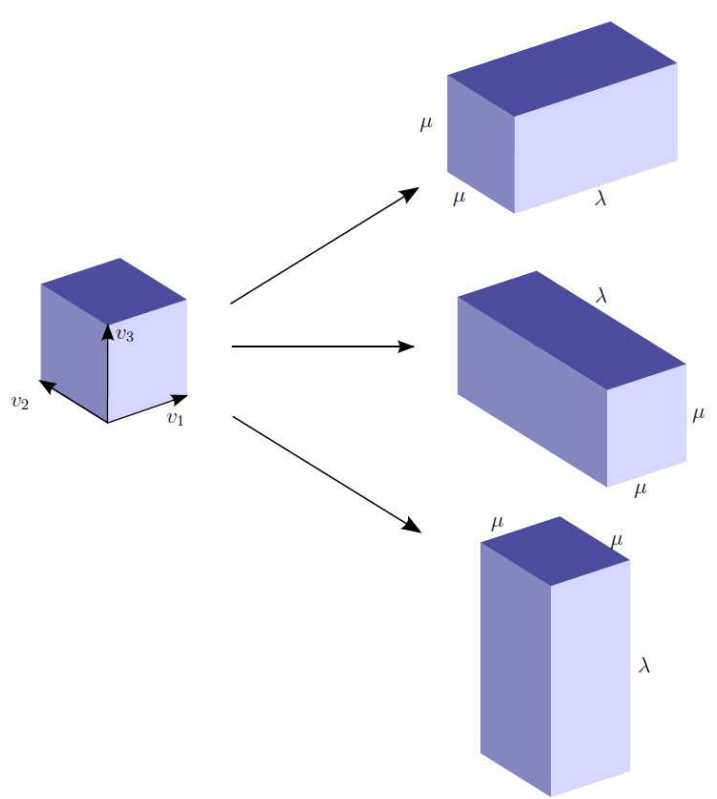

Fig. 1 Cubic-to-tetragonal transformation.

until the stress reaches a critical value $G^{+}$. At that point, phase transformation (from austenite to martensite) starts. An increase of the applied strain results in an increase of the martensitic volume fraction, at the expense of the austenitic volume fraction. The martensitic variants that develop are those which are the most favorably oriented with respect to the applied loading. There ultimately comes a moment when austenite is fully transformed into martensite. At that point, the material behaves elastically again. The unloading stage can be interpreted in a similar way. In particular, the stress plateau at $\sigma=G^{-}$corresponds to the reverse transformation from martensite to austenite.

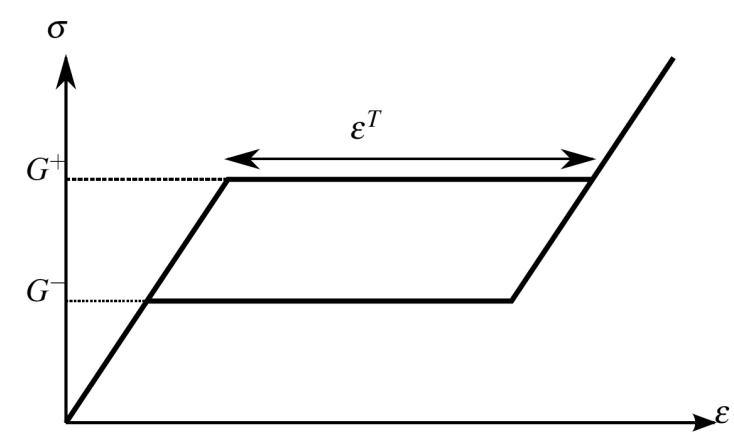

Fig. 2 Superelastic response of shape memory alloys.

Many constitutive models of SMAs have been proposed, both for uniaxial and multi axial loadings. Here we consider models which enter the framework of standard generalized materials [10]. In that framework, the local state of the material is described by the strain $\varepsilon$ and an internal variable $\alpha$. It is essential for our purpose to consider that $\alpha$ is constrained to take values in a given set $\mathscr{K}$ that is not a vectorial space (typically, $\mathscr{K}$ is a bounded set). The behaviour of the material is determined by the free energy function $w(\varepsilon, \alpha)$ and the dissipation potential $\Phi(\dot{\alpha})$ according to the following constitutive relations

$\sigma=\frac{\partial w}{\partial \varepsilon}(\varepsilon, \alpha), A=-\frac{\partial w}{\partial \alpha}(\varepsilon, \alpha)$,

$A=A^{d}+A^{r}, A^{d} \in \partial \Phi(\dot{\alpha}), A^{r} \in \partial I_{\mathscr{K}}(\alpha)$.

In (1)-(2), $\dot{\alpha}$ is the left-time derivative, $A$ is the thermodynamical force associated to $\alpha$, and $\partial$ denotes the subdifferential operator [3]. Let $\mathbb{A}=$ vect $\mathscr{K}$ be the vectorial space spanned by $\mathscr{K}$. We recall that the subdifferential $\partial f$ of a function $f: \mathbb{A} \mapsto \mathbb{R}$ is the multi-valued mapping defined by

$\partial f(x)=\{\tau \in \mathbb{A} \mid f(y)-f(x) \geq \tau .(y-x) \forall y \in \mathbb{A}\}$.

If $f$ is convex, then $\partial f$ is a monotone operator [3], i.e.:

$\left(y^{\prime}-y\right) .\left(x^{\prime}-x\right) \geq 0$ for all $x \in \mathbb{A}, x^{\prime} \in \mathbb{A}, y \in \partial f(x), y^{\prime} \in \partial f\left(x^{\prime}\right)$.

The dissipation potential $\Phi$ is a convex positive function such that $\Phi(0)=0$. In (2), the set $\partial \Phi(0)$ can be interpreted as the elasticity domain of the material, i.e. as the set of thermodynamical forces $A$ compatible with a purely elastic behaviour $(\dot{\alpha}=0)$. The term $A^{r}$ in (2) arises as a consequence of the constraint $\alpha \in \mathscr{K}$. We refer to [6] for a derivation of (1-2) from the principle of thermodynamics.

In this paper, we consider free energy functions $w(\varepsilon, \alpha)$ of the form

$w(\varepsilon, \alpha)=\frac{1}{2}(\varepsilon-K . \alpha): L:(\varepsilon-K . \alpha)+f(\alpha)$

where $L$ is symmetric positive, $K: \mathbb{A} \mapsto \mathbb{E}$ is a linear mapping, and $f: \mathbb{A} \mapsto \mathbb{R}$ is a positive differentiable function (not necessarily linear nor convex). In such case, we have from (1)

$$
\sigma=L:(\varepsilon-K . \alpha), A=K^{t}: \sigma-f^{\prime}(\alpha)
$$

where $K^{t}$ is the transpose of $K$. The total strain $\varepsilon$ is thus the sum of an elastic strain $L^{-1}: \sigma$ (proportional to the stress) and an inelastic strain $K . \alpha$ (proportional to the internal variable $\alpha$ ).

It can easily be verified that the uniaxial response of SMAs pictured in Fig. 2 can be modeled by taking

$$
\begin{aligned}
& \mathscr{K}=\left[0, \varepsilon^{T}\right], \\
& w(\varepsilon, \alpha)=\frac{1}{2} E(\varepsilon-\alpha)^{2}, \\
& \Phi(\dot{\alpha})=\frac{\left(G^{+}-G^{-}\right)}{2}|\dot{\alpha}|+\frac{\left(G^{+}+G^{-}\right)}{2} \dot{\alpha}
\end{aligned}
$$

where $\alpha / \varepsilon^{T}$ can be interpreted as the volume fraction of the martensite. The parameter $\varepsilon^{T}$ corresponds to the strain amplitude of the plateau in Fig. 2 and is interpreted as the maximum strain that can be achieved by phase transformation. 
Observe that the model defined by (6) is almost identical to the uniaxial model of the elastic-perfectly plastic behavior, corresponding to

$$
\mathscr{K}=\mathbb{R}, w(\varepsilon, \alpha)=\frac{1}{2} E(\varepsilon-\alpha)^{2}, \Phi(\dot{\alpha})=\sigma_{y}|\dot{\alpha}|
$$

The only meaningful difference between the two models lies in the constraint $\alpha \in\left[0, \varepsilon^{T}\right]$ that is imposed for SMAs. That constraint has a profound impact on the large-time behavior, as we shall see.

It is well known that the framework of generalized standard materials can be used to derive three-dimensional models of hardening plasticity [15]. Similarly, many existing models of shape-memory alloys can be written in the format considered $[8,17]$. For instance, a three-dimensional micromechanical model of SMA frequently used in the literature [7, $20,19]$ is given by

$$
\begin{aligned}
& w(\varepsilon, \xi)=\frac{1}{2}\left(\varepsilon-\sum_{i=1}^{n} \xi_{i} \varepsilon_{i}\right): L:\left(\varepsilon-\sum_{i=1}^{n} \xi_{i} \varepsilon_{i}\right)+\sum_{i=1} m_{i} \xi_{i}, \\
& \Phi(\dot{\xi})=\sum_{i=1}^{n} \frac{\left(G_{i}^{+}-G_{i}^{-}\right)}{2}\left|\dot{\xi}_{i}\right|+\frac{\left(G_{i}^{+}+G_{i}^{-}\right)}{2} \dot{\xi}_{i} \\
& \mathscr{K}=\left\{\xi \in \mathbb{R}_{+}^{n}: \sum_{i=1}^{n} \xi_{i} \leq 1\right\}
\end{aligned}
$$

where $n$ is the number of martensitic variants and $\varepsilon_{i}$ is the given transformation strain for each variant. The internal variable $\xi=\left(\xi_{1}, \cdots, \xi_{n}\right)$ represents the set of volume fractions for each martensitic variant. Because of mass conservation in the phase transformation, the volume fraction of the austenite is equal to $1-\sum_{i} \xi_{i}$, hence the constraint $\sum_{i} \xi_{i} \leq$ 1 that is imposed on $\xi$. Note that the model (7) is formally quite similar to crystal plasticity with $n$ slip systems. The main difference lies in the constraint that is imposed on $\alpha$. More sophisticated micromechanical models of the free energy have been proposed to improve on (7). In such models, an extra term $f(\alpha)$ is added to the free energy in (7) to account for the geometric incompatibility of the martensitic variants $[8,17]$. That term $f(\alpha)$ is classically refered to as a mixing energy. For instance, the expression of $f(\alpha)$ proposed in [17] is

$$
\begin{aligned}
f(\alpha)=\sup _{a \geq 0 \mid L-K(a) \geq 0} & \frac{1}{2} \sum_{r=1}^{n} \alpha_{r} \varepsilon_{r}: M(a): \varepsilon_{r} \\
& -\frac{1}{2} \sum_{r, s=1}^{n} \alpha_{r} \alpha_{s} \varepsilon_{r}: M(a): \varepsilon_{s} .
\end{aligned}
$$

In (8), the notation $M \geq 0$ is used to indicate that a secondorder symmetric tensor $M$ is positive, i.e. satisfies $u . M . u \geq 0$ for all vectors $u$. Let $\varepsilon^{*}$ denote the adjugate of $\varepsilon=\left(\varepsilon_{i j}\right) \in$ $\mathbb{R}^{3 \times 3}$, defined by the relations

$$
\varepsilon_{i i}^{*}=\varepsilon_{j j} \varepsilon_{k k}-\varepsilon_{j k}^{2}, \varepsilon_{j k}^{*}=\varepsilon_{j i} \varepsilon_{k i}-\varepsilon_{j k} \varepsilon_{i i},
$$

for any $\{i, j, k\}$ permutation of $\{1,2,3\}$. In (8), $K(a)$ is the symmetric fourth-order tensor such that $(1 / 2) \bar{\varepsilon}: K(a): \bar{\varepsilon}=$ $-a: \bar{\varepsilon}^{*}$ for all $\bar{\varepsilon}$, and $M(a)$ is defined by

$M(a)=-K(a)-K(a):(L-K(a))^{-1}: K(a)$.

An important observation is that the mixing energy $f$ is not necessarily convex, as can be verified on (8). Much emphasis has been put on micromechanical SMA models, but we also note that phenomenological SMA models can also enter the format considered [26,2].

In the following we consider a material described by constitutive laws (1-2-5) and occupying a domain $\Omega$. Body forces $f^{d}$ are prescribed in $\Omega$. Displacements $u^{d}$ are imposed on a part $\Gamma_{u}$ of the boundary $\Gamma$, and tractions $T^{d}$ are prescribed on $\Gamma_{T}=\Gamma-\Gamma_{u}$. The functions $f^{d}, u^{d}, T^{d}$ as well as the the stress and state variables $(\sigma, \varepsilon, \alpha)$ depend on $(x, t)$. In order to alleviate the expressions, this dependence will be omitted in the notations, unless in case of possible ambiguities.

Quasi-static evolutions of the continuum are governed by the following system:

$$
\begin{gathered}
\sigma \in \mathscr{K}_{\sigma}, \varepsilon \in \mathscr{K}_{\varepsilon}, \alpha \in \mathscr{K}, \\
A^{d} \in \partial \Phi(\dot{\alpha}), A^{r} \in \partial I_{\mathscr{K}}(\alpha), \\
\sigma=L:(\varepsilon-K . \alpha), \\
K^{t}: \sigma-f^{\prime}(\alpha)=A^{d}+A^{r},
\end{gathered}
$$

where $\mathscr{K}_{\sigma}$ and $\mathscr{K}_{\varepsilon}$ are respectively the sets of admissible stress and strain fields, defined by

$\mathscr{K}_{\sigma}=\left\{\sigma \mid \operatorname{div} \sigma+f^{d}=0\right.$ in $\Omega ; \sigma . n=T^{d}$ on $\left.\Gamma_{T}\right\}$,

$\mathscr{K}_{\varepsilon}=\left\{\varepsilon \mid \varepsilon=\left(\nabla u+\nabla^{t} u\right) / 2\right.$ in $\Omega ; u=u^{d}$ on $\left.\Gamma_{u}\right\}$.

We are interested in finding sufficient conditions on the loading $\left(f^{d}, T^{d}, u^{d}\right)$ for shakedown to occur, in the sense that the dissipated energy remains bounded in time. This is related (but not strictly equivalent) to the fact that the internal variable $\alpha$ tends to a limit as time tends to infinity (see e.g. [15, $18]$ for a more detailed discussion along those lines).

\section{Static shakedown theorem}

It is convenient to introduce the so-called fictitious elastic response $\left(\sigma^{E}, \varepsilon^{E}\right)$ of the system, i.e. the response that would be obtained if the material was purely elastic. More precisely, $\left(\sigma^{E}, \varepsilon^{E}\right)$ is the solution of

$\sigma^{E} \in \mathscr{K}_{\sigma}, \varepsilon^{E} \in \mathscr{K}_{\varepsilon}, \sigma^{E}=L: \varepsilon^{E}$.

The goal of this section is to prove the following

Shakedown theorem for SMAs If there exists $m>1, \tau \geq 0$ and a time-independent field $A_{*}^{r}(x)$ such that

$m K^{t}: \sigma^{E}(x, t)-A_{*}^{r}(x) \in \partial \Phi(0) \forall x \in \Omega, \forall t>\tau$

then there is shakedown, whatever the initial condition is. 
Proof Consider a solution $\left(\varepsilon, \alpha, \sigma, A^{r}, A^{d}\right)$ of the evolution problem (for some given initial condition) and let

$$
D(t)=\int_{\Omega} A^{d} \cdot \dot{\alpha} d x
$$

be the rate of dissipated energy. Assuming $\left(m, \tau, A_{*}^{r}\right)$ satisfy (13), we show in the following that the total dissipated en$\operatorname{ergy} \int_{0}^{T} D(t) d t$ remains bounded as $T \rightarrow \infty$. To that purpose, introduce the functional $W(t)$ defined as

$$
W(t)=\int_{\Omega} w\left(\varepsilon(t)-\varepsilon^{E}(t), \alpha(t)\right) d x
$$

We have

$$
\dot{W}(t)=\int_{\Omega}\left[\left(\sigma-\sigma^{E}\right):\left(\dot{\varepsilon}-\dot{\varepsilon}^{E}-K \cdot \dot{\alpha}\right)+f^{\prime}(\alpha) \cdot \dot{\alpha}\right] d x .
$$

Since $\operatorname{div}\left(\sigma-\sigma^{E}\right)=0$ in $\Omega,\left(\sigma-\sigma^{E}\right) . n=0$ on $\Gamma_{T}$ and $u-u^{E}=0$ on $\Gamma_{u}$, the principle of virtual power gives

$$
\int_{\Omega}\left(\sigma-\sigma^{E}\right):\left(\dot{\varepsilon}-\dot{\varepsilon}^{E}\right) d x=0 .
$$

Therefore

$$
\dot{W}(t)=\int_{\Omega}\left[-K^{t}:\left(\sigma-\sigma^{E}\right)+f^{\prime}(\alpha)\right] \dot{\alpha} d x
$$

which using (10) can be rewritten as

$\dot{W}(t)=-D(t)+\int_{\Omega}\left[-A^{r}+K^{t}: \sigma^{E}\right] \cdot \dot{\alpha} d x$.

Setting $A_{*}^{d}=m K^{t}: \sigma^{E}(t)-A_{*}^{r}$, we obtain

$\dot{W}(t)=-D(t)+\int_{\Omega}\left[-A^{r}+\frac{1}{m}\left(A_{*}^{d}+A_{*}^{r}\right)\right] \cdot \dot{\alpha} d x$.

The property (13) shows that $A_{*}^{d} \in \partial \Phi(0)$ for $t>\tau$. Since $A^{d} \in \partial \Phi(\dot{\alpha})$ and $A_{*}^{d} \in \partial \Phi(0)$, Eq. (4) gives

$\left(A^{d}-A_{*}^{d}\right) \cdot \dot{\alpha} \geq 0$.

Moreover, since $A^{r} \in \partial I \mathscr{K}(\alpha)$ and $\alpha \in \mathscr{K}$, Eq. (3) gives

$$
0 \geq A^{r}(t) .\left(\alpha\left(t^{\prime}\right)-\alpha(t)\right)
$$

for any $t^{\prime}$. Taking the limit $t^{\prime} \longrightarrow t$ with $t^{\prime}<t$, we obtain

$A^{r} . \dot{\alpha} \geq 0$

where we recall that $\dot{\alpha}$ is the left-time derivative. Combining (16)-(17) with (15) yields

$$
\dot{W}(t) \leq \frac{1-m}{m} D(t)+\frac{1}{m} \int_{\Omega} A_{*}^{r} \cdot \dot{\alpha} d x .
$$

Integrating with respect to time on $[\tau, T]$ and noting that $A_{*}^{r}$ is time-independent, we find

$$
(m-1) \int_{\tau}^{T} D(t) d t \leq m W(\tau)+\int_{\Omega} A_{*}^{r} \cdot(\alpha(T)-\alpha(\tau)) d x
$$

where the property $W(T) \geq 0$ has been used. Since $\mathscr{K}$ is bounded, there exists a positive constant $K$ such that $|\alpha| \leq K$ for any $\alpha \in \mathscr{K}$. Therefore

$$
\int_{\Omega} A_{*}^{r} \cdot(\alpha(t)-\alpha(\tau)) d x \leq 2 K \int_{\Omega}\left|A_{*}^{r}\right| d x
$$

Combining that inequality with (18) gives

$$
(m-1) \int_{\tau}^{T} D(t) d t \leq m W(\tau)+2 K \int_{\Omega}\left|A_{*}^{r}\right| d x
$$

The right-hand side of that inequality is independent on $T$. Therefore $\int_{\tau}^{T} D(t)$ is bounded as $T \longrightarrow+\infty$, which completes the proof.

As an illustration, consider the micromechanical model of SMA given by (7). From the above theorem, a sufficient condition for shakedown is that there exists $m>1, \tau \geq 0$ and time-independent scalars $A_{i}(x)$ such that

$$
m \varepsilon_{i}: \sigma^{E}(x, t)-A_{i}(x) \in\left[G_{i}^{-}, G_{i}^{+}\right]
$$

for all $t>\tau, x \in \Omega$ and $i=1, \cdots, n$. That condition is equivalent to

$$
m\left(\sup _{t} \varepsilon_{i}: \sigma^{E}(x, t)-\inf _{t} \varepsilon_{i}: \sigma^{E}(x, t)\right) \leq G_{i}^{+}-G_{i}^{-}
$$

for all $i=1, \cdots, n, x \in \Omega$ and $t>\tau$. Therefore the condition for shakedown is that there exists some $m$ such that

$1<m \leq \frac{G_{i}^{+}-G_{i}^{-}}{\sup _{t} \varepsilon_{i}: \sigma^{E}(x, t)-\inf _{t} \varepsilon_{i}: \sigma^{E}(x, t)}$

for all $i=1, \cdots, n$ and $x \in \Omega$. The obtained shakedown condition is merely a restriction on the local amplitude of the loading.

We note that the obtained condition for shakedown is independent on the function $f$ that appears in the free energy (5). Moreover, it can be observed that the proof detailed above does not require the function $f$ to be convex. Therefore, the condition for shakedown (19) actually applies for the micromechanical models with non convex mixing energies that have been discussed previously.

It is insightful to compare the obtained theorem with the classical Melan's theorem for Elastic-Perfectly plastic (abbreviated as EP in the following) materials, which corresponds to $f=0$ and $\mathscr{K}=\mathbb{A}$ :

Shakedown theorem for EP materials (Melan) If there exists $m>1, \tau \geq 0$ and a time-independent self-stress field $\rho_{*}^{r}(x)$ such that

$m K^{t}: \sigma^{E}(x, t)-\rho_{*}^{r}(x) \in \partial \Phi(0) \forall x \in \Omega, \forall t>\tau$

then there is shakedown, whatever the initial condition is. 
In (20), self-stress fields $\rho$ are characterized by the relations

$\operatorname{div}\left(\sigma-\sigma^{E}\right)=0$ in $\Omega,\left(\sigma-\sigma^{E}\right) . n=0$ on $\Gamma_{T}$

The statement of the SMA shakedown theorem is formally similar to that of the classical Melan's theorem. There is a crucial difference, however: the field $\rho_{*}$ in (20) is submitted to the differential constraint (21), whereas the field $A_{*}^{r}$ in (13) is free from any constraint. Phase-transformation can thus only improve the shakedown limit, in the sense that if there exists $\rho_{*}$ satisfying (20), then Eq. (13) is also satisfied by $A_{*}^{r}=\rho_{*}$ (the reverse is generally not true).

\section{Study of a three-bar truss-structure}

We consider the three-bar truss structure represented in Fig. 3 . The structure is submitted to a vertical time-varying force $P(t)$ applied at point $M$ (see Fig.3). The bars have the same cross-sectional area $A$ and are free to rotate at both extremities (pinned connections). The length of the middle bar (labelled as bar 1) is $l_{1}=l$. The lengths of the two other bars (labelled as bars 2 and 3) are $l_{2}=l_{3}=l / \cos \theta$ where $\theta \in$ $[0, \pi / 2]$ is the angle between bar 1 and bar 2 . The constitutive law in each bar is

$\sigma_{i}=E\left(\varepsilon_{i}-\alpha_{i}\right)$

$\sigma_{i} \in \partial \Phi\left(\dot{\alpha}_{i}\right)+\partial I_{\mathscr{K}}\left(\alpha_{i}\right)$

where $\sigma_{i}, \varepsilon_{i}, \alpha_{i}$ are respectively the uniaxial stress, the total uniaxial strain and the inelastic strain in bar $i$. In (22), the constant $E$ is the Young's modulus.

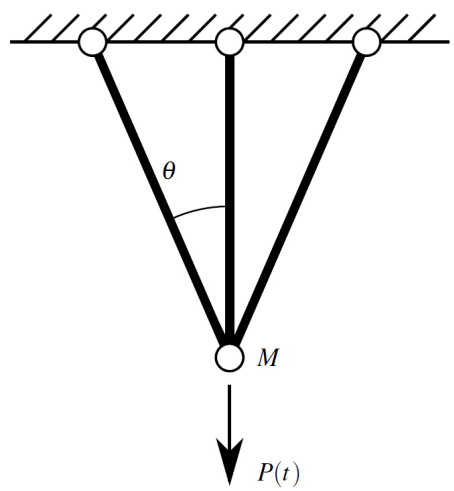

Fig. 3 Three-bar truss.

In (23), the set $\mathscr{K}$ of admissible values for the variable $\alpha_{i}$ is taken as

$\mathscr{K}=\left[-\varepsilon^{T}, \varepsilon^{T}\right]$ where $\varepsilon^{T}>0$ is fixed. The limiting case $\varepsilon^{T}=+\infty$ corresponds to plasticity. The dissipation potential in (23) is taken in the form

$\Phi(\dot{\alpha})=\frac{\sigma_{y}}{n}|\dot{\alpha}|^{n}$

where $n \geq 1$ is given. Therefore the elasticity domain $\partial \Phi(0)$ of the material is equal to $\left[-\sigma_{y}, \sigma_{y}\right]$. In the case of plasticity, $\sigma_{y}$ can be interpreted as the yield limit. The special case $n=1$ corresponds to a rate-independent behavior.

In the following we restrict our attention to evolutions that respect the symmetry of the structure, i.e. such that

$$
\sigma_{2}(t)=\sigma_{3}(t), \varepsilon_{2}(t)=\varepsilon_{3}(t), \alpha_{2}(t)=\alpha_{3}(t) .
$$

In such condition, the equilibrium implies that

$\sigma_{1}(t)+2 \sigma_{2}(t) \cos \theta=p(t)$

where $p(t)=P(t) / A$. Under the assumption of infinitesimal strains, the geometric compatibility of the deformations in the bars implies that

$\varepsilon_{2}(t)=\varepsilon_{1}(t) \cos ^{2} \theta$

The fictitious elastic response $\sigma^{E}(t)$ of the structure, obtained by solving (22-26-27) with $\alpha_{i}=0$, is given by

$\sigma_{1}^{E}(t)=\frac{p(t)}{1+2 \cos ^{3} \theta}, \sigma_{2}^{E}(t)=\frac{p(t) \cos ^{2} \theta}{1+2 \cos ^{3} \theta}$.

From (26), residual stress states $\rho$ satisfy the relation

$\rho_{1}+2 \rho_{2} \cos \theta=0$

where $\rho_{i}$ is the residual stress in bar $i$.

\subsection{Case $\varepsilon^{T}=\infty$}

Let us first consider the plastic case $\left(\varepsilon^{T}=+\infty\right)$. Using Melan's theorem, we obtain that shakedown occurs (independently on the initial state) if there exists $\left(\rho_{1}, \rho_{2}\right)$ satisfying (29) and such that

$\left|\rho_{i}+\sigma_{i}^{E}(t)\right|<\sigma_{y}$

for all $t$. The condition (30) sets restrictions on the values taken by $p(t)$. Using the expressions (28) and (29), the condition (30) can indeed be rewritten as

$-1<p^{\prime}(t)-2 \rho \cos \theta<1$,

$-1<p^{\prime}(t) \cos ^{2} \theta+\rho<1$

where $\rho=\rho_{2} / \sigma_{y}$ and

$p^{\prime}(t)=\frac{p(t)}{\sigma_{y}\left(1+2 \cos ^{3} \theta\right)}$. 
The condition (31) needs to be satisfied for all $t$ by some time-independent $\rho$. Let $p_{\text {min }}=\inf _{t} p(t)$ and $p_{\text {max }}=\sup _{t} p(t)$. We set

$$
p_{\text {min }}^{\prime}=\frac{p_{\min }}{\sigma_{y}\left(1+2 \cos ^{3} \theta\right)}, p_{\text {max }}^{\prime}=\frac{p_{\max }}{\sigma_{y}\left(1+2 \cos ^{3} \theta\right)} .
$$

The requirement (31) is equivalent to

$$
\begin{aligned}
& -1<p_{\text {min }}^{\prime}-2 \rho \cos \theta ; p_{\text {max }}^{\prime}-2 \rho \cos \theta<1 ; \\
& -1<p_{\text {min }}^{\prime} \cos ^{2} \theta+\rho ; p_{\text {max }}^{\prime} \cos ^{2} \theta+\rho<1 ;
\end{aligned}
$$

i.e. to $m\left(p_{\min }, p_{\max }\right)<\rho<M\left(p_{\min }, p_{\max }\right)$ with

$$
\begin{aligned}
& m\left(p_{\text {min }}, p_{\text {max }}\right)=\max \left(\frac{p_{\text {max }}^{\prime}-1}{2 \cos \theta},-1-p_{\text {min }}^{\prime} \cos ^{2} \theta\right), \\
& M\left(p_{\text {min }}, p_{\text {max }}\right)=\min \left(\frac{p_{\text {min }}^{\prime}+1}{2 \cos \theta}, 1-p_{\text {max }}^{\prime} \cos ^{2} \theta\right) .
\end{aligned}
$$

There exists $\rho$ satisfying (31) if and only if

$m\left(p_{\min }, p_{\max }\right)<M\left(p_{\min }, p_{\max }\right)$.

That last inequality is a sufficient condition on $p_{\min }$ and $p_{\max }$ for shakedown to occur. As usual in shakedown analysis, the condition for shakedown does not depend on the exact time-dependence of the loading $p(t)$ : only the extreme values $p_{\text {min }}$ and $p_{\text {max }}$ are needed.

The condition (34) can be made explicit in some special situations. Let us first consider the case $p_{\text {min }}=0$. We are interested in finding the values of $p_{\max }$ verifying (34). The condition (34) is found to be satisfied for

$$
p_{\max }<\sigma_{y}(1+2 \cos \theta) .
$$

The value $\sigma_{y}(1+2 \cos \theta)$ actually corresponds to the plastic limit load for the structure. Let us now consider the case $p_{\max }=-p_{\min }>0$. The condition (34) can then be rewritten as

$$
\max \left\{-\alpha\left(p_{\max }^{\prime}\right),-\beta\left(p_{\max }^{\prime}\right)\right\}<\min \left\{\alpha\left(p_{\max }^{\prime}\right), \beta\left(p_{\max }^{\prime}\right)\right\}
$$

where

$$
\alpha\left(p_{\max }^{\prime}\right)=\left(1-p_{\max }^{\prime}\right) / 2 \cos \theta
$$

and

$$
\beta\left(p_{\text {max }}^{\prime}\right)=1-p_{\text {max }}^{\prime} \cos ^{2} \theta .
$$

The condition (35) can readily be seen to hold if and only if

$\alpha\left(p_{\max }^{\prime}\right)>0, \beta\left(p_{\text {max }}^{\prime}\right)>0$.

Using the expression of $\alpha\left(p_{\max }^{\prime}\right)$ and $\beta\left(p_{\max }^{\prime}\right)$, the requirement (36) is found to be equivalent to $p_{\max }^{\prime}<1$, i.e.

$$
p_{\max }<\sigma_{y}\left(1+2 \cos ^{3} \theta\right) .
$$

The obtained shakedown limits, represented as solid lines in Fig. 4, are decreasing functions of the angle $\theta$.

\subsection{Case $\varepsilon^{T}<+\infty$}

We now consider that $\varepsilon^{T}<+\infty$ and use the theorem presented in Sec. 3 to determine the shakedown limit. For a set $\mathscr{K}$ given by (24), the subgradient $\partial I_{\mathscr{K}}$ can reach any value in $\mathbb{R}$, i.e.

$$
\cup_{y \in \mathbb{R}} \partial I_{\mathscr{K}}(y)=\mathbb{R} .
$$

We thus obtain from Sec. 3 that shakedown occurs if there exists some time-independent $A_{i}$ such that

$\left|A_{i}+\sigma_{i}^{E}(t)\right|<\sigma_{y}$

for all $t$. The condition (37) looks similar to the condition (30) obtained previously for the case of plasticity. However, in contrast with the values $\rho_{i}$ in (30), the values $A_{i}$ in (37) are not submitted to any constraint. Therefore, the condition (37) translates to a restriction on the amplitude of $\sigma_{i}^{E}(t)$, namely

$$
\sup _{t} \sigma_{i}^{E}(t)-\inf _{t} \sigma_{i}^{E}(t)<2 \sigma_{y} .
$$

The expression (28) of $\sigma_{i}^{E}$ shows that most severe restriction is obtained for $i=1$ and can be rewritten as

$$
p_{\max }^{\prime}-p_{\min }^{\prime}<2 \text {. }
$$

In the case $p_{\min }=0$, we thus obtain that the shakedown limit corresponds to $p_{\max }^{\prime}=2$, i.e. $p_{\max }=2 \sigma_{y}\left(1+2 \cos ^{3} \theta\right)$.

In the case $p_{\max }=-p_{\min }>0$, the shakedown limit is $\sigma_{y}\left(1+2 \cos ^{3} \theta\right)$, as for the plastic case.

The obtained shakedown limits are represented as dotted lines in Fig. 4.

For $p_{\text {min }}=0$, the shakedown limit for the SMA material is strictly above the shakedown limit for the EP material. This is a situation where constraints on the internal variable have a beneficial effect on the shakedown behavior. For $p_{\text {min }}=-p_{\text {max }}$, the shakedown limit for phase transformation coincides with the shakedown limit for plasticity. However, there are still significant differences between the asymptotic behaviour of those two materials, as explained in the next section.

\section{Asymptotic behavior under cyclic loadings}

We consider a cyclic loading $p(t)=p_{\max } \sin (2 \pi t / T)$ with $p_{\text {max }}>\sigma_{y}\left(1+2 \cos ^{3} \theta\right)$, i.e. beyond the shakedown limit of the SMA and the EP materials. We compare the response of the structure for the case $\varepsilon^{T}=+\infty$ and $\varepsilon^{T}<+\infty$, using numerical step-by-step analysis.

For an elastic-plastic material, the plastic strain rate $\dot{\alpha}_{i}$ is known to converge towards a cyclic stabilized response $\dot{\alpha}_{i}^{\infty}(t)$. There is either alternating plasticity if $\int_{0}^{T} \dot{\alpha}_{i}^{\infty}(t) d t=$ 0 , or ratchetting (i.e. incremental collapse) otherwise. A remarkable feature of elastic-plastic materials is that the type of asymptotic regime is independent on the initial state [9]. 


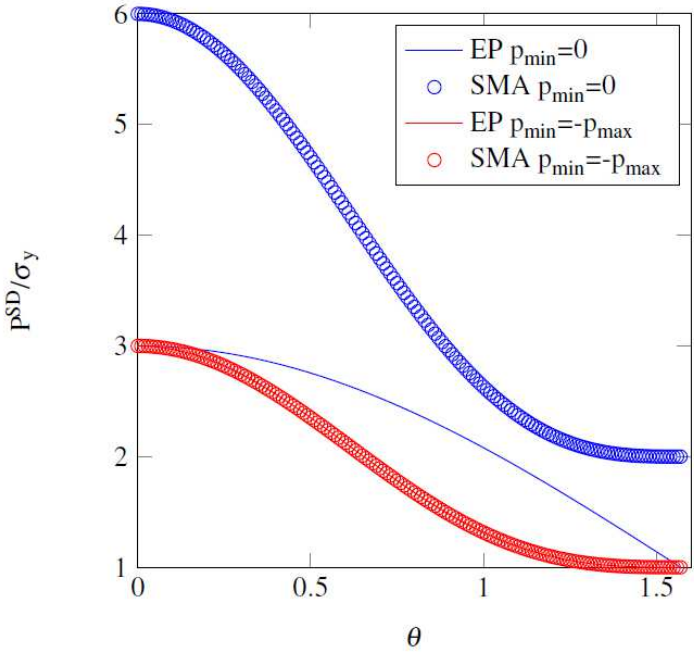

Fig. 4 Shakedown limits for the elastic-plastic material (EP) and for shape memory alloys (SMA) (the continuous red lines and the red symbols are perfectly superimposed).

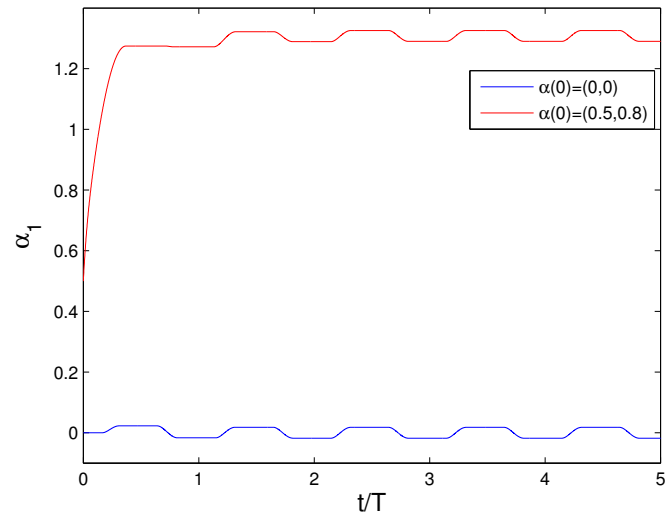

Fig. 5 Evolutions of $\alpha_{1}$ for two different initial states, case $\varepsilon^{T}=\infty$.

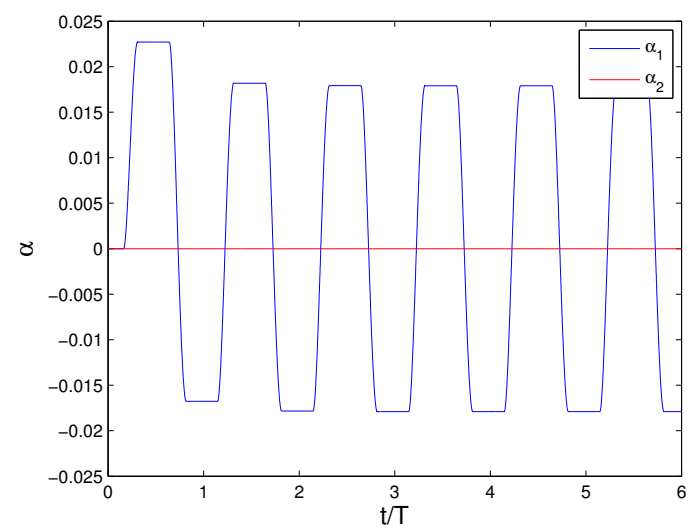

Fig. 6 Evolutions of $\alpha$ for the initial condition $\alpha(0)=(0,0)$, case $\varepsilon^{T}=1, p_{\max }=2 \sigma_{y}$.

In contrast, the behaviour of the SMA material is found to be more complex. Numerical simulations indeed reveal

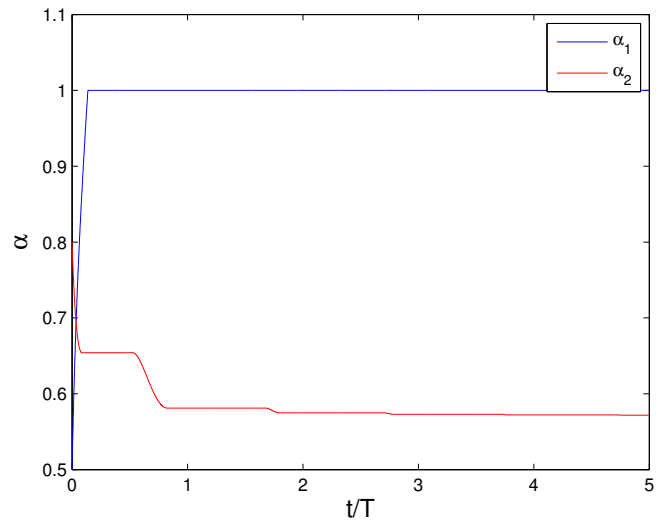

Fig. 7 Evolutions of $\alpha$ for the initial condition $\alpha(0)=(0.5,0.8)$, case $\varepsilon^{T}=1, p_{\max }=2 \sigma_{y}$.

that there is still convergence towards a cyclic response $\dot{\alpha}_{i}^{\infty}(t)$. However, the stabilized values $\dot{\alpha}_{i}^{\infty}(t)$ as well as the asymptotic regime may depend on the initial state considered.

Those considerations are illustrated in Figs 5-7. For the elastic-plastic material, Fig. 5 shows the evolution of the plastic strain $\alpha_{1}$ obtained from two different initial conditions. Values of the parameters are $\sigma_{y} / E=0.1, n=2, \theta=$ $\pi / 4, p_{\max }=2 \sigma_{y}$. The initial states considered are given by $\alpha(0)=(0,0)$ and $\alpha(0)=(0.5,0.8)$. For the elastic plastic material, we do observe an alternating plasticity behaviour. Moreover, the stabilized plastic strain response in the two evolutions only differ by a constant, which illustrates the uniqueness of the plastic strain rates in the asymptotic regime. Fig. 6-7 shows the evolution of $\alpha_{i}$ for the SMA material, using the same data as in Fig 5. For $\alpha(0)=(0,0)$, we observe the convergence of $\alpha_{i}$ towards a cyclic but non constant response, akin to alternating plasticity. Such a behavior is referred to as alternating phase transformation in the following. For the initial state $\alpha(0)=(0.5,0.8)$, a shakedown behaviour is actually observed even though the shakedown limit provided by the theorem of Sec. 3 is exceeded. There is no contradiction: the theorem gives a limit for shakedown to occur whatever the initial state is. In the present case, shakedown occurs for some but not all initial condition. We note that such a behavior cannot exist in standard plasticity.

The path dependence of the asymptotic regime arises as a consequence of the constraint $\alpha_{i} \in[-1,1]$, and was also observed in another example for which analytical solutions can be obtained [18]. This rather intriguing behaviour rises the issue of finding how the asymptotic regime varies with the initial state. In the language of dynamical systems, this consists in finding the basins of attractions for the different types of asymptotic behaviour. Such results are shown in Fig 8-10. Those figures give a map of the asymptotic regime (alternating phase transformation (AP) or shakedown (SD)) as a function of the initial state $\alpha(0)$. Ratchetting is obviously 
excluded since the internal variable $\alpha_{i}$ is physically constrained to lie in $[-1,1]$. As can be seen in Fig. 8-10, there is a region surrounding $(1,1)$ and $(-1,-1)$ for which shakedown occurs. The basins of attraction depend on $p_{\text {max }}$. In particular, the basin of attraction for shakedown get smaller as $p_{\max }$ increases, and is found to vanish for $p_{\max }>2.5 \sigma_{y}$.

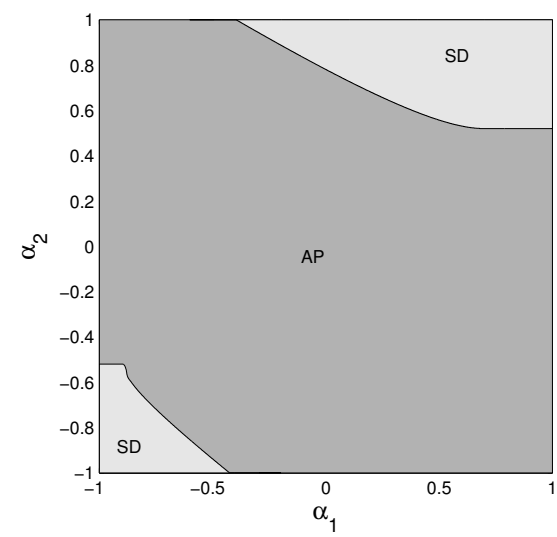

Fig. 8 Map of the asymptotic regime, $p_{\max }=2 \sigma_{y}$.

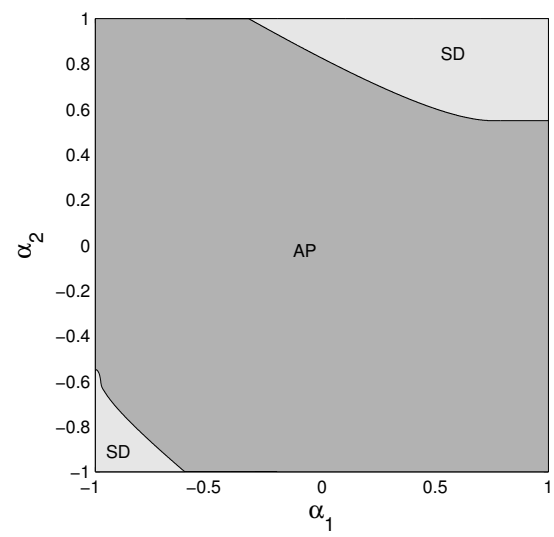

Fig. 9 Map of the asymptotic regime, $p_{\max }=2.42 \sigma_{y}$.

An other point of interest is the asymptotic behaviour of the dissipated energy per cycle, defined as

$$
\Delta=\int_{\Omega} \Delta(x) d \omega
$$

where

$$
\Delta(x)=\lim _{n \rightarrow+\infty} \int_{n T}^{(n+1) T} \sigma: \dot{\alpha} d t .
$$

The local quantity $\Delta(x)$ is indeed related to the low-cycle fatigue lifetime of the material [4]: the fatigue lifetime typically decreases as $\Delta(x)$ increases. In the case of shape memory alloys, the value of $\Delta$ depends on the initial state considered. This is reflected in Fig. 11, which shows the mini-

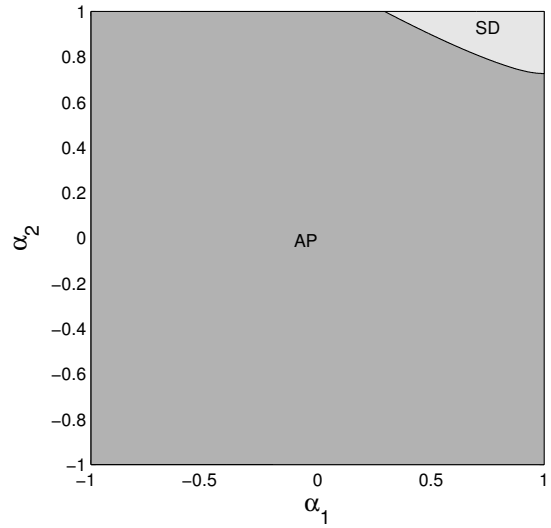

Fig. 10 Map of the asymptotic regime, $p_{\max }=2.43 \sigma_{y}$.

mum and maximum values of $\Delta$ over a sample of 100 initial states $\alpha(0)=\left(\alpha_{1}(0), \alpha_{2}(0)\right)$ (equidistributed in the domain $[-1,1] \times[-1,1])$. For each initial state, $\Delta$ is estimated numerically by solving the evolution equations over $20 \mathrm{cy}-$ cles, which proved to be sufficient for reaching the asymptotic state with a good accuracy. The minimum and maximum values of $\Delta$ over the samples considered are denoted by $\min \Delta$ and $\max \Delta$.

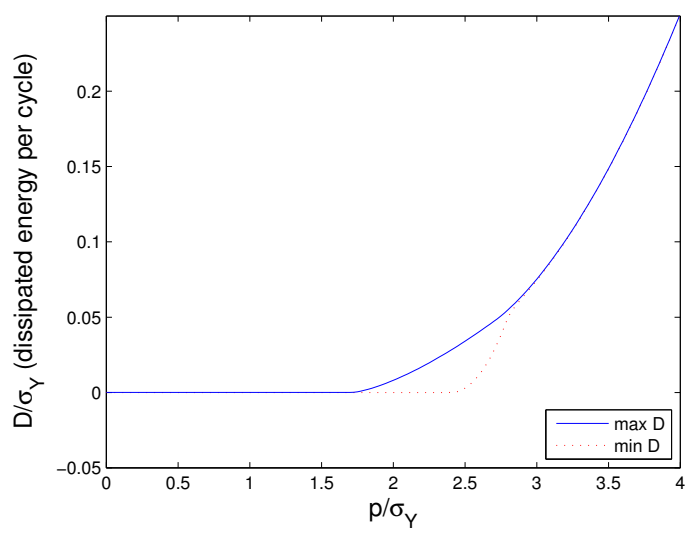

Fig. 11 Dissipated energy on the stabilized cycle.

For $p \leq \sigma_{y}\left(1+2 \cos ^{3} \theta\right)$, we obtain that $\min \Delta=\max \Delta=$ 0 . This is in agreement with the results of the shakedown theorem: as long as $p \leq \sigma_{y}\left(1+2 \cos ^{3} \theta\right)$, the asymptotic behavior is elastic and accordingly $\Delta$ is equal to 0 , whatever the initial state is. For $\sigma_{y}\left(1+2 \cos ^{3} \theta\right) \leq p \leq 2.5$, we obtain that $\min \Delta=0$ and $\max \Delta>0$ : some initial conditions lead to shakedown whereas some others lead to alternating phase transformation, in accordance with the maps in Fig. 8-10.

For $2.5<p / \sigma_{y}<3$, we have $0<\min D<\max D$ : all initial condition lead to alternating phase transformation, but the stabilized energy dissipation remains dependent on the initial state considered. An interesting observation is that, 
for $p / \sigma_{y}>3$, the curves $\max \Delta$ and $\min \Delta$ are found to coincide (up to the accuracy of the computations). This is an indication of the fact that the asymptotic value of $\Delta$ becomes independent on the initial state. In such condition, the lowcycle fatigue lifetime is also expected to be independent on the initial state, as in the plastic case. The asymptotic rates $\dot{\sigma}$ and $\dot{\alpha}$ are also found to be independent on the initial state.

The plots on Fig. 8-11 correspond to $\theta=\pi / 4$, but similar properties are observed for all values of $\theta$. This leads us to distinguish between three domains in a diagram $(\theta, p)$ (Fig. 12). The first one is the shakedown domain given by $|p| \leq \sigma_{y}\left(1+2 \cos ^{3} \theta\right)$. The second domain is defined by $0<\min \Delta=\max \Delta$ and corresponds to large values of $|p|$, for which the large-time energy dissipation $\Delta$ is non zero and path independent. The third domain is defined by the condition $\min \Delta<\max \Delta$ and corresponds to intermediate values of $p$ for which the asymptotic regime depends on the initial state. That last domain would vanish in standard plasticity. Whereas the exact expression of the shakedown domain is predicted by the theorem of Sec. 3, the domain $0<\min \Delta=\max \Delta$ is only obtained numerically. It would be interesting to see if that domain could be predicted by theoretical analysis.

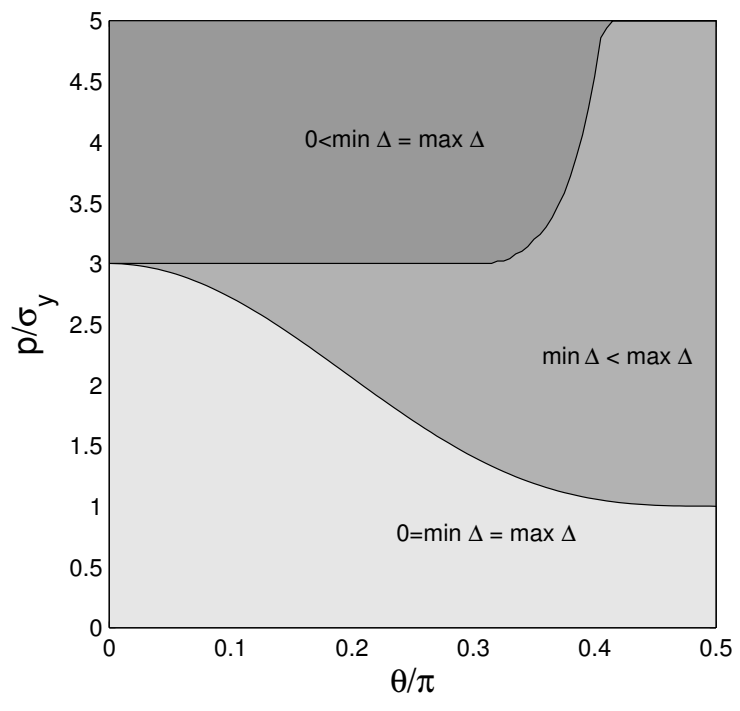

Fig. 12 Behaviour of the stabilized energy dissipation.

\section{Concluding remarks}

The shakedown behavior of SMA structures has been discussed in this paper. The shakedown theorem presented in Sec. 3 gives a sufficient condition for the energy dissipation to remain bounded in time, independently on the initial state. That theorem applies to a wide range of constitutive models of SMAs. When the loading exceeds the limit provided by that theorem, the asymptotic regime may depend on the initial state of the structure: in contrast with plasticity, some initial condition may lead to shakedown whereas some others may lead to alternating phase transformation. For the example presented in Sec. 4, however, numerical simulations suggest that path-independence of the energy dissipation is recovered when the loading is large enough. It is not clear whether that property is general or just a specific feature of the example considered.

We note that all this rather complex behavior is a consequence of the constraint $\alpha \in \mathscr{K}$ that is imposed on the internal variable. Comparing the shakedown theorem of Sec. 3 with the classical Melan's theorem shows that phase transformation can only have a beneficial influence on the shakedown limit, contrary to [5]. That conclusion, however, should be tempered by the fact that phase-transformation and plasticity should actually be considered as two coexisting processes. Studying the shakedown behavior of coupled plasticity / phase-transformation models is the object of future work. Although some results in that direction have been provided in [18], a lot of progress remains to be made.

Acknowledgements Part of this work has been motivated by discussions with Dr.-Ing. J.W. Simon during the Euromech Colloquium 'Direct and variational methods for non smooth problems in mechanics' (Amboise, 24-26 June 2013), organized by Pr. G. De Saxcé et Pr. G. Del Piero.

\section{References}

1. Akel, S., Nguyen, Q.: Determination of the cyclic response in cyclic plasticity. In: Owen, D.R.J. et al. (Eds), Computational Plasticity: Models, Software and Applications, Pineridge Press, Swansea (1989)

2. Auricchio, F., Petrini, L.: A three-dimensional model describing stress-temperature induced solid phase transformations: solution algorithm and boundary value problems. Int.J.Num.Meth.Eng. 61, 807-836 (2004)

3. Brézis, H.: Opérateurs maximaux monotones et semigroupes de contractions dans les espaces de hilbert. North-Holland, Amsterdam (1972)

4. Constantinescu, A., Dang Van, K., Maitournam, M.: A unified approach for high and low cycle fatigue based on shakedown concepts. Eng. Mater. Struct. 26(6), 561-568 (2003)

5. Feng, X., Sun, Q.: Shakedown analysis of shape memory alloy structures. Int J. Plasticity 23, 183-206 (2007)

6. Frémond, M.: Non-smooth thermomechanics. Springer (2002)

7. Govindjee, S., Miehe, C.: A multi-variant martensitic phase transformation model: formulation and numerical implementation. Comput. Meth. Appl. Mech. Engrg. 191, 215-238 (2001)

8. Hackl, K., Heinen, R.: An upper bound to the free energy of $n$-variant polycrystalline shape memory alloys. J.Mech.Phys.Solids 56, 2832-2843 (2008)

9. Halphen, B.: Accommodation et adaptation des structures élastoviscoplastiques et plastiques. Association amicale des ingénieurs anciens élèves de l'ENPC (1978)

10. Halphen, B., Nguyen, Q.S.: Sur les matériaux standards généralisés. J.Mécanique 14, 1-37 (1975) 
11. Koiter, W.T.: General problems for elastic solids. Progress in solid mechanics (1960)

12. Kružík, M., Mielke, A., Roubícek, T.: Modelling of microstructure and its evolution in shape-memory alloy single crystals, in particular in cualni. Meccanica 40, 389-418 (2005)

13. Maitournam, H., Pommier, B., Thomas, J.J.: Détermination de la réponse asymptotique d'une structure anélastique sous chargement cyclique. C.R.Mecanique 330, 703-708 (2002)

14. Melan, E.: Theorie statisch unbestimmter systeme aus idealplastischen baustoff. Sitz.Berl.Ak.Wiss. 145, 195-218 (1936)

15. Nguyen, Q.S.: On shakedown analysis in hardening plasticity. J.Mech.Phys.Solids 51, 101-125 (2003)

16. Peigney, M.: A time-integration scheme for thermomechanical evolutions of shape-memory alloys. C.R.Mecanique 334(4), 266271 (2006)

17. Peigney, M.: A non-convex lower bound on the effective free energy of polycrystalline shape memory alloys. J.Mech.Phys.Solids 57, 970-986 (2009)

18. Peigney, M.: Shakedown theorems and asymptotic behaviour of solids in nonsmooth mechanics. Eur. J. Mech. A 29, 785793 (2010)

19. Peigney, M., Seguin, J.: An incremental variational approach to coupled thermo-mechanical problems in anelastic solids. application to shape-memory alloys. Int. J. Sol. Struct. 50(24), 40434054 (2013)

20. Peigney, M., Seguin, J., Hervé-Luanco, E.: Numerical simulation of shape memory alloys structures using interior-point methods. Int. J. Sol. Struct. 48(20), 2791-2799 (2011)

21. Peigney, M., Stolz, C.: Approche par contrôle optimal des structures élastoviscoplastiques sous chargement cyclique. C.R.Acad.Sci. Paris, Série II 329, 643-648 (2001)

22. Peigney, M., Stolz, C.: An optimal control approach to the analysis of inelastic structures under cyclic loading. J.Mech.Phys.Solids 51, 575-605 (2003)

23. Pham, D.: On shakedown theory for elastic-plastic materials and extensions. J.Mech.Phys.Solids 56, 1905-1915 (2008)

24. Simon, J.W.: Direct evaluation of the limit states of engineering structures exhibiting limited, nonlinear kinematical hardening. Int J. Plasticity 42, 141-167 (2013)

25. Simon, J.W., Weichert, D.: Shakedown analysis of engineering structures with limited kinematical hardening. Int. J. Sol. Struct. 49(15), 2177-2186 (2012)

26. Souza, A., Mamiya, E., Zouain, N.: Three-dimensional model for solids undergoing stress-induced phase transformations. Eur.J.Mech. A 17, 789-806 (1998)

27. Spiliopoulos, K.V., Panagiotou, K.D.: A direct method to predict cyclic steady states of elastoplastic structures. Comput. Methods Appl. Mech. Engrg. 223, 186-198 (2012)

28. Wesfreid, E.: Etude du comportement asymptotique pour un modèle viscoplastique. C.R.Acad.Sci.Paris A 290, 297-300 (1980)

29. Zarka, J., Frelat, J., Inglebert, G.: A new approach to inelastic analysis of structures. Martinus Nijhoff Publishers, Dordrecht (1988) 